\title{
Thin-Stratified Medium Fast-Multipole Algorithm (TSMFMA) for Solving 2.5D Microstrip Structures
}

\author{
Jie L. Xiong* and Weng Cho Chew \\ The University of Hong Kong and University of Illinois at \\ Urbana-Champaign \\ E-mail: jxiong2@uiuc.edu
}

\section{Introduction}

Microstrip structures are planar or quasi-planar metallic structures that reside on or straddle across thin-stratified medium. Thin-stratified medium fast-multipole Algorithm (TSMFMA) [1] has been proposed for this type of stuctures and has greatly reduced the cost of microstrip structures' analysis. However, the previous algorithm is limited to the analysis of planar structures only. In order to apply it to the structures that straddle across substrates, such as microstrip patch with vertical coaxial feed or planar inverted-F antennas (PIFA), we need to modify the algorithm and improve the flexibility of it.

In this paper, we started with a newly developed formula of layered medium Green's function, which is naturally decomposed into $\mathrm{TE}_{z}$ and $\mathrm{TM}_{z}$ parts. By detouring the integration path of the Green's function from Sommerfeld integration path (SIP) to the vertical branch cut and including the contribution from any pertinent poles, the original integration can be reduced to the summation of a finite number of terms, involving only the zeroth order Hankel function. Then we decompose the propagation factor to functions of $z$ or $z^{\prime}$ only, and apply TSMFMA to the decomposed form. In this manner, the structures with $z$-variations could be analyzed without much additional complexity.

\section{Formulation}

Recently, our group has proposed an alternative and elegant way of deriving layered medium Green's function[2]. The dyadic Green's function for layered medium can be expressed as

$$
\begin{aligned}
\overline{\mathbf{G}}\left(\mathbf{r}, \mathbf{r}^{\prime}\right) & =(\nabla \times \hat{\mathbf{z}})\left(\nabla^{\prime} \times \hat{\mathbf{z}}\right) g^{\mathrm{TE}}\left(\mathbf{r}, \mathbf{r}^{\prime}\right) \\
& +\frac{1}{k_{n m}^{2}}(\nabla \times \nabla \times \hat{\mathbf{z}})\left(\nabla^{\prime} \times \nabla^{\prime} \times \hat{\mathbf{z}}\right) g^{\mathrm{TM}}\left(\mathbf{r}, \mathbf{r}^{\prime}\right),
\end{aligned}
$$

where $k_{n m}^{2}=\omega^{2} \epsilon_{n} \mu_{m}$ ( $m$ and $n$ are indexes of the layers which contain the source point $\mathbf{r}^{\prime}$ and field point $\mathbf{r}$ respectively). And

$$
\begin{aligned}
g^{\mathrm{TE}}\left(\mathbf{r}, \mathbf{r}^{\prime}\right) & =\frac{i}{4 \pi} \int_{0}^{\infty} \frac{d k_{\rho}}{k_{m z} k_{\rho}}\left[J_{0}\left(k_{\rho} r_{s}\right) F^{\mathrm{TE}}\left(k_{\rho}, z, z^{\prime}\right)\right], \\
g^{\mathrm{TM}}\left(\mathbf{r}, \mathbf{r}^{\prime}\right) & =\frac{i}{4 \pi} \int_{0}^{\infty} \frac{d k_{\rho}}{k_{m z} k_{\rho}}\left[J_{0}\left(k_{\rho} r_{s}\right) F^{\mathrm{TM}}\left(k_{\rho}, z, z^{\prime}\right)\right] .
\end{aligned}
$$

$F^{\mathrm{TM}}$ and $F^{\mathrm{TE}}$ describe the propagation of $B_{z}$ or $D_{z}$ fields for TE and TM fields in 
an inhomogeneous layer. This formulation contains only two basic integrals for TE and TM waves and involves only zeroth order Bessel function. We have presented the five-term matrix element representation derived from this form in [2]. However, for the application of thin-stratified medium fast-multipole algorithm (TSMFMA), it is better to start with Equation (1).

By using the fact that $J_{0}\left(k_{\rho} \rho\right)=1 / 2\left[H_{0}^{(1)}\left(k_{\rho} \rho\right)+H_{0}^{(2)}\left(k_{\rho} \rho\right)\right]$ and the reflection formula that $H_{0}^{(1)}(-x)=-H_{0}^{(2)}(x)$, we get

$$
g^{a}\left(\mathbf{r}, \mathbf{r}^{\prime}\right)=\frac{i}{8 \pi} \int_{-\infty}^{\infty} \frac{d k_{\rho}}{k_{m z} k_{\rho}}\left[H_{0}^{(1)}\left(k_{\rho} r_{s}\right) F^{a}\left(k_{\rho}, z, z^{\prime}\right)\right]
$$

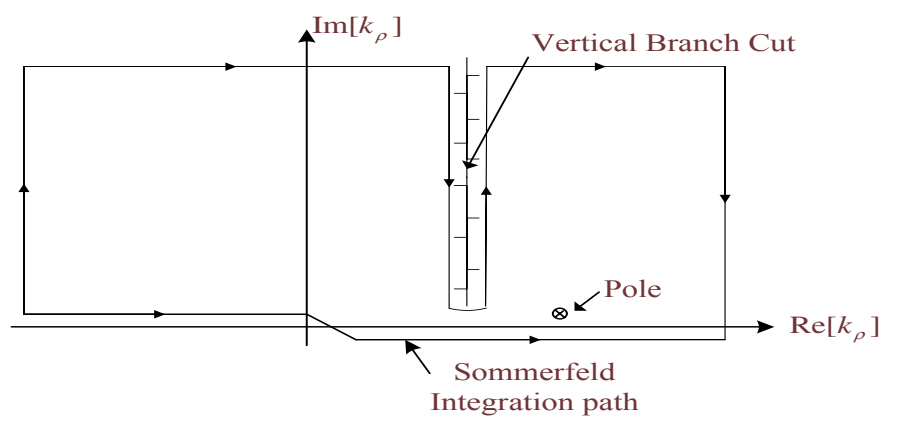

Figure 1: Deformation of the Sommerfeld integration path to the vertical branch cut

From reference [1], we can detour the integration path to the vertical branch cut as in Fig. 1. The original integration is decomposed into the contribution from the vertical branch cut and the enclosed poles. By performing a numerical integration along the vertical branch cut, the integration becomes

$$
\begin{aligned}
g^{a}\left(\mathbf{r}, \mathbf{r}^{\prime}\right)= & \frac{i}{8 \pi} \sum_{p=1}^{N_{q}} w_{p} \frac{\delta k_{\rho, p}}{k_{m z, p} k_{\rho, p}}\left[H_{0}^{(1)}\left(k_{\rho, p} r_{s}\right) F^{a}\left(k_{\rho, p}, z, z^{\prime}\right)\right] \\
& +\frac{i}{8 \pi} \sum_{p=1}^{N_{p}} 2 \pi i \operatorname{Res}\left[F^{a}\left(k_{\rho, p}, z, z^{\prime}\right)\right] \frac{1}{k_{m z, p} k_{\rho, p}} H_{0}^{(1)}\left(k_{\rho, p} r_{s}\right)
\end{aligned}
$$

where the $N_{q}$ is the number of quadrature points and $N_{p}$ is the number of poles.

Decomposing the Hankel function by the same techniques as in 2D MLFMA,

$$
\begin{aligned}
& \overline{\mathbf{G}}^{T E}\left(\mathbf{r}, \mathbf{r}^{\prime}\right)=(\nabla \times \hat{z})\left(\nabla^{\prime} \times \hat{z}\right) g^{T E}\left(\mathbf{r}, \mathbf{r}^{\prime}\right) \\
= & \frac{i}{16 \pi^{2}}(\nabla \times \hat{z})\left(\nabla^{\prime} \times \hat{z}\right) \sum_{p=1}^{N_{r}} \frac{v_{p}}{k_{m z, p} k_{\rho, p}} \int_{0}^{2 \pi} d \alpha \tilde{\beta}_{j l^{\prime}}(\alpha) \tilde{\alpha}_{l^{\prime} l}(\alpha) \tilde{\beta}_{l i}(\alpha) F_{r e v}^{T E}(z) F_{r a d}^{T E}\left(z^{\prime}\right) \\
= & \frac{i}{8 \pi N_{a}} \sum_{k=1}^{N_{a}} \sum_{p=1}^{N_{r}} \frac{v_{p}}{k_{\rho, p}} \underbrace{\left[(\nabla \times \hat{z}) \tilde{\beta}_{j l^{\prime}}\left(\alpha_{k}\right) F_{r e v}^{T E}(z)\right]}_{\text {receiving pattern }} \tilde{\alpha}_{l^{\prime} l}\left(\alpha_{k}\right) \underbrace{\left[\left(\nabla^{\prime} \times \hat{z}\right) \tilde{\beta}_{l i}\left(\alpha_{k}\right) \frac{F_{r a d}^{T E}\left(z^{\prime}\right)}{k_{m z, p}}\right]}_{\text {radiation pattern }}
\end{aligned}
$$


where $v_{p}=w_{p} \delta k_{\rho, p}$ for quadrature points and $v_{p}=2 \pi i \operatorname{Res}\left[F^{\alpha}\right]$ for poles.

Similarly, the TM part of the dyadic Green's function can be decomposed as

$$
\begin{gathered}
\overline{\mathbf{G}}^{T M}\left(\mathbf{r}, \mathbf{r}^{\prime}\right)=\left(\frac{1}{\epsilon_{r, n}} \nabla \times \nabla \times \hat{z}\right)\left(\nabla^{\prime} \times \nabla^{\prime} \times \hat{z}\right) g^{T M}\left(\mathbf{r}, \mathbf{r}^{\prime}\right) \\
=\frac{i}{8 \pi N_{a}} \sum_{k=1}^{N_{a}} \sum_{p=1}^{N_{r}} \frac{v_{p}}{k_{\rho, p}} \underbrace{\left[\frac{(\nabla \times \nabla \times \hat{z})}{\epsilon_{r, n}} \tilde{\beta}_{j l^{\prime}}\left(\alpha_{k}\right) F_{r e v}^{T M}(z)\right]}_{\text {receiving pattern }} \\
\cdot \tilde{\alpha}_{l^{\prime} l}\left(\alpha_{k}\right) \underbrace{\left[\left(\nabla^{\prime} \times \nabla^{\prime} \times \hat{z}\right) \tilde{\beta}_{l i}\left(\alpha_{k}\right) \frac{F_{r a d}^{T M}\left(z^{\prime}\right)}{k_{m z, p}}\right]}_{\text {radiation pattern }}
\end{gathered}
$$

If the field point and source point lie in different layers, only two pairs of radiation patterns and receiving patterns are needed, one for TM wave and the other for TE wave. For example, if the field point is above the source point $\left(z>z^{\prime}, n>m\right)$,

$$
\begin{gathered}
I_{r a d, m}^{T E}=\frac{1}{k_{m z}} \int d \mathbf{r}^{\prime}\left[\left(\mathbf{k}_{\rho, p k} \times \hat{z}\right) \cdot \mathbf{J}_{i}\left(\mathbf{r}^{\prime}\right)\right] e^{-i \mathbf{k}_{\rho, p k} \cdot\left(\rho_{l}-\rho_{i}\right)} \\
C_{m}\left[e^{i k_{m z}\left(d_{m+1}-z^{\prime}\right)}+G_{m, m-1} e^{i k_{m z}\left(d_{m+1}+z^{\prime}-2 d_{m}\right)}\right] \\
I_{r e v, n}^{T E}=\int d \mathbf{r}\left[\left(\mathbf{k}_{\rho, p k} \times \hat{z}\right) \cdot \mathbf{J}_{T j}(\mathbf{r})\right] e^{-i \mathbf{k}_{\rho, p k} \cdot\left(\rho_{j}-\rho_{l}\right)} \\
D_{m n}\left[e^{i k_{n z}\left(z-d_{n}\right)}+G_{n, n+1} e^{i k_{n z}\left(2 d_{n+1}-z-d_{n}\right)}\right]
\end{gathered}
$$

where $G_{m, n}$ is the generalized reflection coefficient for TE wave, $C_{m}, D_{m, n}$ are functions of the reflection coefficients (see [3] for detailed expressions). The radiation and receiving patterns for TM wave or other cases can be derived similarly. When the field and source point belong to the same layer, three pairs of radiation and receiving patterns for each wave mode are needed, two of which represent the reflected terms and one for the direct term. Moreover, the treatment of the direct term needs some extra manipulation. The propagation factor represents the direct term is $\exp \left(i k_{0}\left|\mathbf{r}-\mathbf{r}^{\prime}\right|\right)$ which cannot be decomposed into $F(z) F\left(z^{\prime}\right)$ simply, thus it is improper to use in the fast algorithm. In order to remove the modulus function, we transform the integral to the $k_{z}$ space.

$$
\begin{aligned}
g^{d}\left(\mathbf{r}, \mathbf{r}^{\prime}\right) & =\frac{i}{8 \pi} \int_{-\infty}^{\infty} \frac{d k_{\rho}}{k_{m z} k_{\rho}} H_{0}^{(1)}\left(k_{\rho} r_{s}\right) e^{i k_{m z}\left|z-z^{\prime}\right|}, \quad d k_{z}=-\frac{k_{\rho}}{k_{z}} d k_{\rho} \\
& =\frac{i}{8 \pi} \int_{-\infty}^{\infty} \frac{d k_{m z}}{k_{\rho}^{2}} H_{0}^{(1)}\left(k_{\rho} r_{s}\right) e^{i k_{m z}\left(z-z^{\prime}\right)}
\end{aligned}
$$

Then we can apply the same technique of detouring the integral and decompose the integrand to implement the TSMFMA to the direct term.

In the derivation of the algorithm, there exists a problem that the two integrals in Equations (2) and (3) are not well defined, because there is a pole at $k_{\rho}=0$. This 
would lead to questions about the legitimacy of the following steps. Here is our argument for this question: first, the pole at $k_{\rho}=0$ is a pseudo pole because when we add contribution from TE and TM wave together, the pole will be cancelled. This can be verified from the matrix element expression in [1]. In another word, in our manipulation of $g^{T E}$ and $g^{T M}$, we have deliberately left the pole alone, because we know the two singularities will cancel each other. Our numerical tests support this argument.

\section{Numerical Results}

A microstrip patch anntenna printed on a dielectric substrate with a vertical coaxial feed connecting to the ground is analyzed. We apply TSMFMA to this structure and compared the results with that obtained from the method of moments using the same Green's function. The two results agree very well.
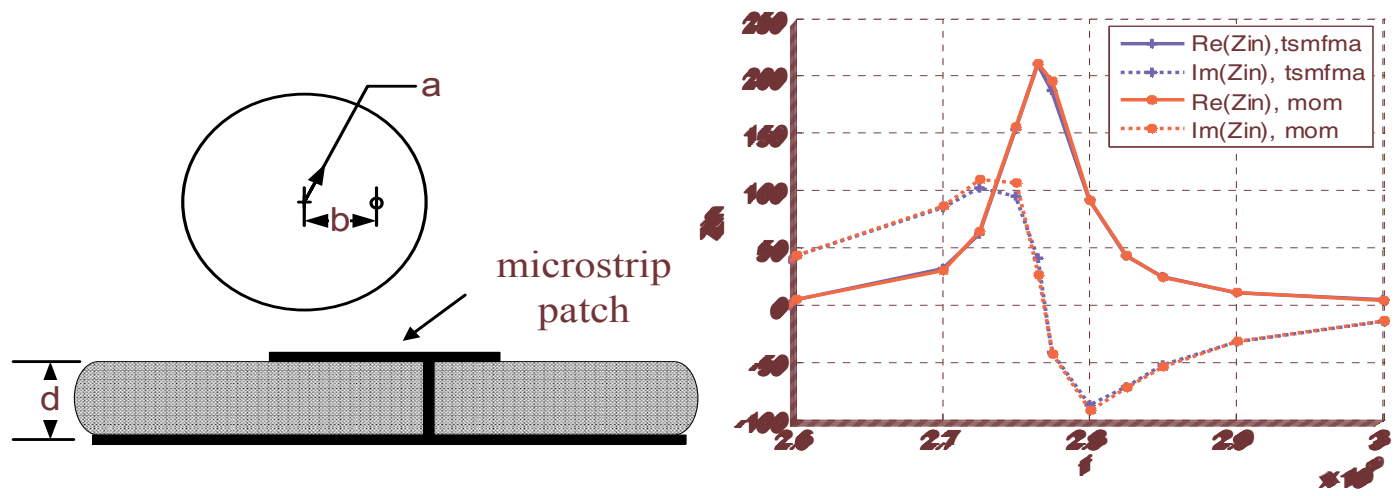

Figure 2: Input impedance of a microstrip patch antenna. $\mathrm{d}=0.16 \mathrm{~cm}, \mathrm{a}=1.88 \mathrm{~cm}$, $\mathrm{b}=0.84 \mathrm{~cm}, \epsilon_{r}=2.6$.

\section{Conclusion}

With the enhanced TSMFMA, we can apply this fast algorithm to quasi-3D objects in layered medium. It improves the flexibility of the original algorithm and uses only a small set of radiation and receiving pattern pairs.

\section{References}

[1] J.S. Zhao, W.C. Chew, C.C. Lu, E. Michielssen and J.M. Song, "Thin-Stratified Medium Fast-Multipole Algorithm for Solving Microstrip Structures", IEEE Trans. Microwave Theory Tech., vol. 46, No. 4, April 1998.

[2] W.C. Chew, J.L. Xiong, and M. A. Saville, "A Matrix-Friendly Formulation of Layered Medium Green's Function", IEEE Antennas Wireless Propagat. Lett., vol. 5, no. 1, pp. 490-494, Dec. 2006.

[3] W.C. Chew, Waves and Fields in Inhomogeneous Media, IEEE Press, 1995 